in vivo $31: 1197-1202(2017)$

doi:10.21873/invivo.11190

\title{
Circulating Tumor Cells as an Auxiliary Diagnostic Tool in Surgery
}

\author{
KATARINA KOLOSTOVA ${ }^{1}$, ADAM RZECHONEK ${ }^{2}$, JAN SCHÜTZNER ${ }^{3}$, ROBERT GRILL ${ }^{1}$, \\ ROBERT LISCHKE ${ }^{3}$, PAVEL HLADIK ${ }^{3}$, JAN SIMONEK ${ }^{3}$ and VLADIMIR BOBEK ${ }^{1,2,3,4}$ \\ ${ }^{1}$ Department of Laboratory Genetics, Kralovske Vinohrady University Hospital, Prague, Czech Republic; \\ ${ }^{2}$ Department of Histology and Embryology, Wroclaw Medical University, Wroclaw, Poland; \\ ${ }^{3}$ Third Department of Surgery, First Faculty of Medicine Charles University \\ in Prague and Motol University Hospital, Prague, Czech Republic \\ ${ }^{4}$ Department of Thoracic Surgery, Masaryk's Hospital in Ustinad Labem, Labem, Czech Republic
}

\begin{abstract}
Background: In general, the presence of circulating tumor cells (CTCs) in peripheral blood $(P B)$ is associated with a relative shorter overall survival in cancer patients. The clinical utility of CTC diagnostics is changing: from prognostic test to an assay predicting therapy response, enabling the right choice of therapy and monitoring the effect of administered therapy. We present two case reports of patients with suspicion of lung and pancreatic cancer, without obtainable preoperative biopsy for histological verification. The focus of the presented study was not to deliver a complete tumor tissue classification to the surgeon, but to answer the question if there is malignant disease or not. The results are based on CTC presence and characterization. Materials and Methods: A size-based separation method for viable CTC enrichment from anticoagulated $P B$ was used. The separated cells were cytomorphologically examined using vital fluorescent microscopy. Additionally, to confirm the epithelial origin of the cells on the separation membrane, CTC gene expression analysis was performed. Results: CTCs were successfully enriched and cultured in vitro in both tested samples. The epithelial character of the captured cells was confirmed by quantitative-polymerase chain reaction ( $q P C R)$ analysis for a set of tumor-associated genes. Conclusion: Detection of cancer cells in PB (liquid biopsy) and their molecular
\end{abstract}

This article is freely accessible online.

Correspondence to: Vladimir Bobek, MD, Ph.D., University Hospital, Kralovske Vinohrady, Department of Laboratory Genetics, Srobarova 50, 10034 Prague, Czech Republic. Tel: +420 26716 3578,e-mail: vbobek@centrum.cz

Key Words: CTC, surgery, MetaCell, pancreatic cancer, lung cancer, liquid biopsy. characterization could significantly help complete the tumor diagnostic process in a time-efficient manner.

Circulating tumor cells (CTCs) are rare cells originating from solid tumors and circulating in the blood stream. CTCs have been known since the nineteenth century, but only recently has development of modern technologic platforms enabled reliable capture and characterization of CTCs. It is now widely accepted that CTCs found in peripheral blood (PB) are involved in the process of hematogenous metastatic spread. CTCs migrate to distant sites for the establishment of secondary foci of disease, but should be mentioned that CTCs are ultimately rare (1-4). Approximately one cancer cell per 100 million cells can be detected in the bloodstream (5). Only some CTCs have the potential to establish metastatic growth after seeding. The new metastatic niche has to support their survival and clonal proliferation. The migrating cells may have undergone epithelial-mesenchymal transition (EMT), and acquired stem cell characteristics enabling self-renewal (5). These may be the cause of metastatic cancer cells becoming relatively resistant to standard chemotherapy (6). Whether, and where, a CTC succeeds in establishing a metastasis depends on its molecular profile, which is not necessarily the same as that found in the primary tumor.

The presence of CTCs in PB is in general associated with a relatively shorter overall survival (1-5, 7-9). Capturing CTCs from blood has been assigned as a 'liquid biopsy'. Driven by technical progress, both CTC enumeration and molecular characterization are expected to provide additional clinical information on prognosis, therapy choice, and effectiveness, as well as drug resistance (10).

Over time, opinions on the clinical utility of CTC diagnostics have changed: from a prognostic test to an assay predicting therapy response, enabling the correct choice of therapy and monitoring the effect of administered therapy. 
Recently, a role for CTC testing has been seen in screening and diagnostics of primary cancer. But the question still remains: How can liquid biopsy help in surgical praxis? The focus of this study was not to deliver complete tumor tissue classification to the surgeon, but to answer the question as to whether there was malignant disease or not based on CTC presence and characterization. The process of CTC testing may help in cases where other methods of obtaining tumor tissue (e.g. tissue biopsy) are not possible.

This study presents two case reports of patients with suspicion of lung and pancreatic cancer, without obtainable preoperative biopsy for histological verification.

\section{Materials and Methods}

Patients. The diagnostic process for two patients with changes detected on computed tomographic (CT) scan is presented in Figures 1 and 2.

Patient I: This patient was 63 years old smoker, with changes detected via CT $(60 \times 4.0 \mathrm{~mm})$ in the upper lobe of the left lung. Repeated bronchoscopy and sputum cultivation showed no malignant cells presence. Transthoracic puncture was not possible because of the tumor localization (Table I, Figure 1).

Patient II: This patient was 67 years old non-smoker, with a 2month history of digestive discomfort. Changes on CT were detected in the head of the pancreas (diameter $180 \mathrm{~mm}$ ). Biopsy of the tissue via endoscopy did not detect any cancer cells (Table I, Figure 2).

CTC enrichment and culture. PB was collected prior to planned surgery. For each patient, approximately $2 \times 8 \mathrm{ml}$ of venous PB was drawn from the antecubital veins and placed into S-Monovette tubes (Sarstedt AG \& Co., Numbrecht, Germany) containing $1.6 \mathrm{mg}$ EDTA $/ \mathrm{ml}$ blood as an anticoagulant. The samples were processed at room temperature using an isolation procedure completed within 24 hours of the blood withdrawal.

A size-based separation method for viable CTC enrichment from anticoagulated PB was used (MetaCell ${ }^{\circledR}$, Ostrava, Czech Republic). The separation process is based on the filtration of PB through porous polycarbonate membrane (pores of $8 \mu \mathrm{m}$ diameter). Blood transfer into the filtration tube was carried out in several steps in order to prevent blood clotting on the membrane filter. The membrane filter was transferred into a 6 -well cultivation plate. RPMI media was then added to the filter top and CTCs cultured directly on the membrane in vitro, under standard cancer cell culture conditions $\left(37^{\circ} \mathrm{C}\right.$ and $5 \%$ atmospheric $\left.\mathrm{CO}_{2}\right)$. The performance of the captured cells during in vitro culturing was monitored by inverted microscopy at any time without restrictions. The CTCs were grown in an FBS-enriched RPMI medium (10\%) ïn short-term culture (35 days). Subsequently they were analyzed by means of fluorescence microscopy. Viable CTCs were observed under a fluorescence microscope applying vital nuclear stain $\left(\mathrm{NucBlue}^{\mathrm{TM}}\right)$ and vital cytoplasmic stain (Celltracker ${ }^{\mathrm{TM}}$; both Life Technologies, Bleiswijk, the Netherlands) (see Figure 3). The figures obtained by microscopy remain archived for additional cytomorphological analysis if needed; cells captured on the membrane were further used in molecular testing. CTCs were lysed in solution and stored at $-20^{\circ} \mathrm{C}$ for later use.

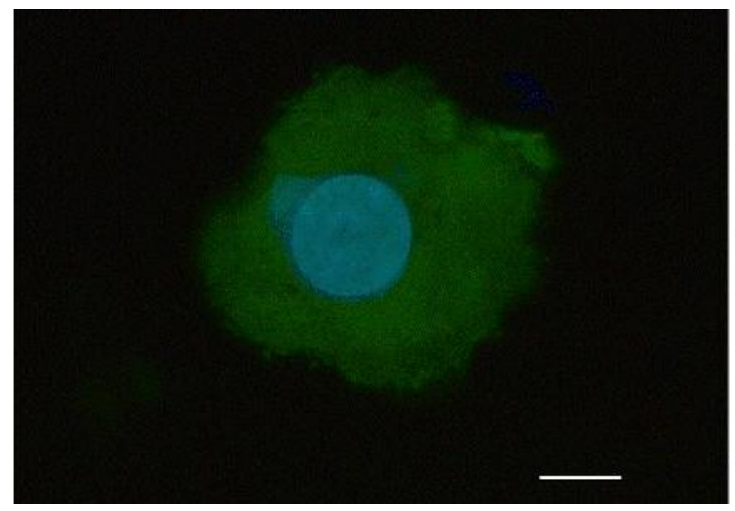

Figure 1. Computer tomography of patient I.

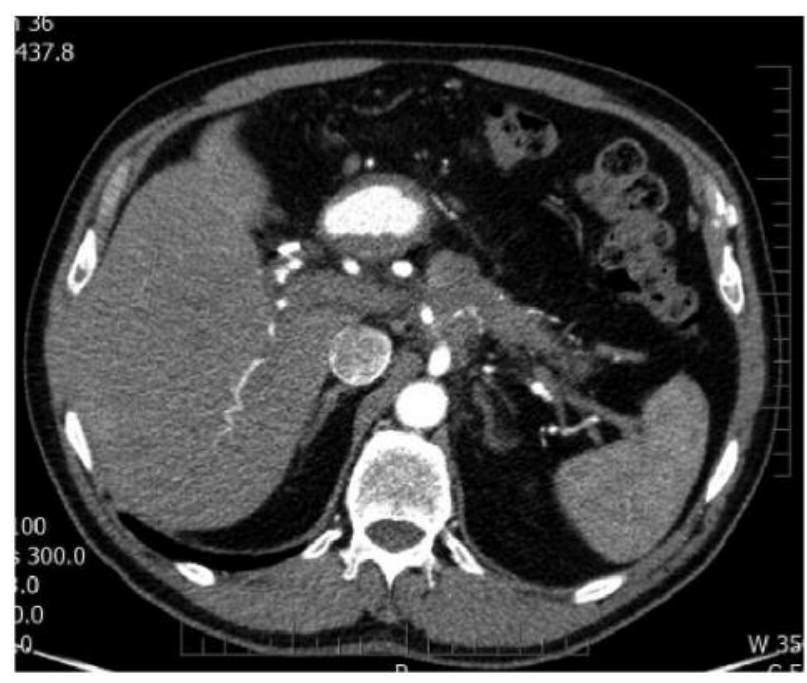

Figure 2. Computer tomography of patient II.

Cytomorphological analysis. Membranes with stained cells were examined using light and fluorescence microscopy in two steps: (i) screening at $\times 20$ magnification to locate cells; (ii) observation at $\times 40-60$ magnification for detailed cytomorphological analysis. Isolated cells and clusters of cells of interest (whether stained or not) were selected, digitized, and examined by an experienced researcher and pathologist. CTCs were defined as cells meeting the following criteria: (i) cell size $\geq 15 \mu \mathrm{m}$ (ii) nuclear size $\geq 10 \mu \mathrm{m}$ ); (iii) irregular nuclear contour; (iv) presence of visible cytoplasm; (v) high nucleus-to-cytoplasm (N/C) ratio (the ratio may change during in vitro culture; (vi) prominent nucleoli; (vii) proliferation activity; (viii) formation of 3D cell layers.

Gene expression analysis (GEA). To confirm the origin of the cells on the separation membrane, CTC gene expression analysis was performed. GEA was conducted in a single quantitative polymerase chain reaction (qPCR) run to assess up to 20 tumor-associated markers expression levels. RNA isolated from different cell 
A

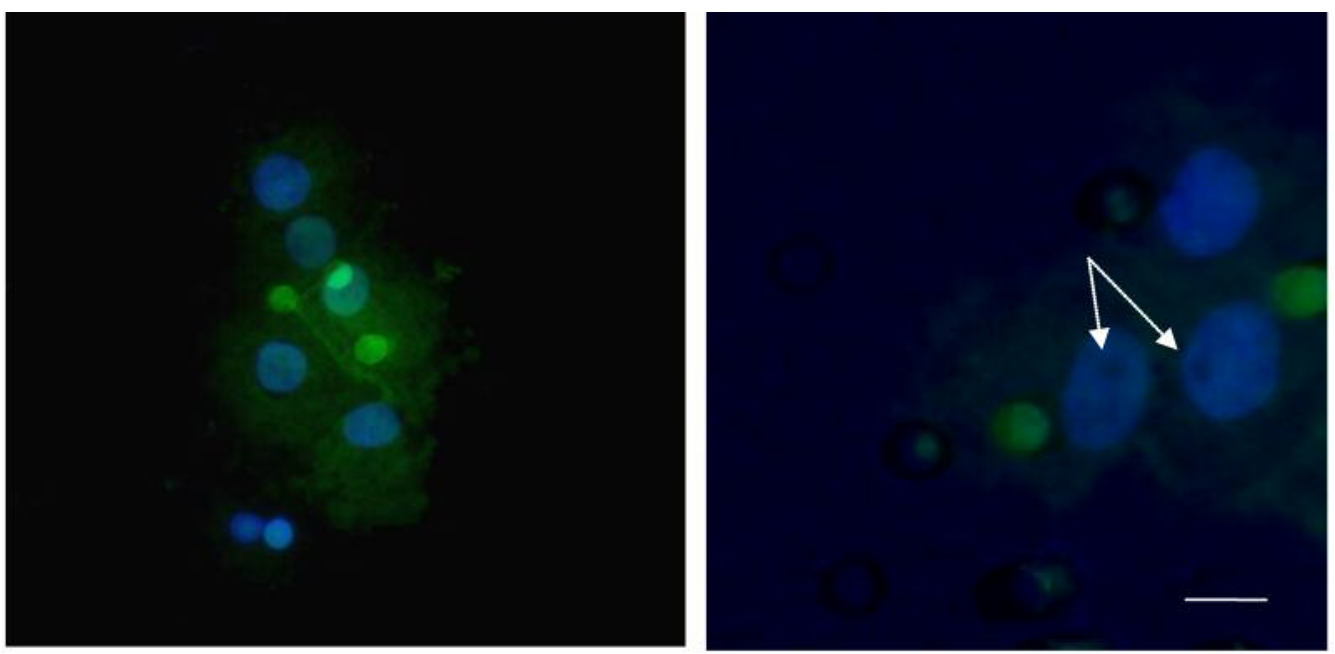

B

Figure 3. Circulating tumor cells (CTCs) isolated from blood of patient I with suspicion of lung cancer (A) and patient II with suspicion of pancreatic cancer $(B)$. CTCs were visualized by vital fluorescent stains (NucBlue ${ }^{T M}$ for nucleus and CellTracker ${ }^{T M}$ for cytoplasm) after short-term in vitro culture (3 days). A: Captured cells were large $(>15 \mu \mathrm{m})$, with large nuclei $(>10 \mu \mathrm{m})$ and exhibited prominent nucleoli (see arrows). B: Captured cells were large $(>15 \mu \mathrm{m})$, with large nuclei $(>10 \mu \mathrm{m})$ and with irregular nuclear contours. Bar represents $10 \mu \mathrm{m}$.

Table I. Characterization of tested patients.

\begin{tabular}{lcc}
\hline & Patient I & Patient II \\
\hline Type of cancer according to CT & Lung cancer (Pancoast tumor) & Pancreatic cancer \\
Age, years & 63 & 69 \\
Tissue examination by: & Bronchoscopy $(\times 2$, negative) & Endoscopy (negative) \\
& Fine-needle biopsy - impossible & Biopsy via endoscopy (negative) \\
\hline
\end{tabular}

fractions (e.g. white blood cells, CTC-enriched fraction, CTCcultured fraction) was compared. Differential diagnostics markers for qPCR test were chosen in concordance with the suspected diagnosis (11-12). For the presented patient cases, the following genes were tested: Actin beta (ACTB), protein tyrosine phosphatase receptor type $\mathrm{C}$ polypeptide/leucocyte common antigen (CD45), macrophage antigen CD68 (CD68), mucin 1 (MUC1), thyroid transcription factor 1 (TTF1), keratin 5 (KRT5), keratin 6 (KRT6), keratin 7 (KRT7), keratin 19 (KRT19), vimentin (VIM), neural cell adhesion molecule 1 (CD56), beta-1,3-glucuronyltransferase 1 
(CD57), carcino embryonic antigen (CEA), synaptophysin. All of the chosen genes have a role in the standard differential diagnostic processes in pathology. A personalized set of tested genes for every patient is shown in Table II. Tested genes are indicated by a X-sign in column "Genes tested" and genes with relatively elevated expression are indicated by a X-sign in column "Genes elevated".

A crucial point of the GEA was to compare the gene expression of the tumor-associated markers in the CTC-enriched fractions to the gene expression of these markers in the white blood cell (WBC) fraction obtained from whole blood.

RNA was isolated from WBC and CTC-enriched fraction on the membrane. The RNA was isolated from the two CTC-fraction types: First, RNA from CTC-enriched fraction obtained immediately after the separation process (these are so-called "virgin CTCs") was isolated, second, RNA from the CTC-enriched fraction grown on the separation membrane in vitro (so called "membrane fraction") was isolated. Some of the cells grown on the membrane in vitro overgrew the membrane and set up a new cell culture on the culturewell bottom. These cells were analyzed as "bottom fraction".

CTC gene expression analysis reported the relative amount of RNA for tumor- associated markers in the WBC fraction and CTCenriched fractions.

All of the cell types were lysed by RLT-buffer with beta-mercaptoethanol (Qiagen). Then, RNA was isolated using RNeasy Mini Kit (Qiagen). The RNA from whole blood cells (WBC fraction) was isolated with a modified RNeasy Mini Kit protocol. The quality and concentration of RNA was measured by NanoDrop (ThermoScientific). As there were only a few hundred cells captured on the membrane, the median concentration of RNA in tested samples was quite low (5-10 $n g / \mu l)$. For cDNA production, the High Capacity cDNA Reverse Transcription Kit (Life Technologies) was used. For quantitative polymerase chain reaction (qPCR) Taqman chemistry including Taqman MGB - probes for all the aforementioned genes (Life Technologies) was employed. The qPCR results were reported as $\mathrm{Cq}$ values. All Cq values were normalized to the normalizer (ACTB gene) and subsequently the normalized values were compared between WBC and CTC fractions for each gene and each patient individually. A modification of standard $\Delta \Delta \mathrm{Cq}$ method was used.

\section{Results}

Patient I. After cytomorphological analysis of captured cells on the separation membrane (Figure 3A) it was concluded that some of them exhibited cancerous characteristics (cell size $>15 \mu \mathrm{m}$, nucleus size $>10 \mu \mathrm{m}$, prominent nucleoli, layer formation). To confirm the origin of the cells, we analyzed gene expression of tumor-associated genes which were chosen due to the rules of differential diagnosis for lung cancer mass in pathology (ACTB, CD45, CD68, MUC1, TTF1, KRT5, KRT6, KRT7, KRT19, VIM, CD56, CD57, $C E A$, synaptophysin). Fifteen genes were tested in total. As reported in Table II, MUC1, TTF 1, KRT7 and KRT19 were identified as being relatively highly expressed in the CTC fraction in comparison to the WBC fraction from whole blood. It was concluded that the combination of $M U C 1$, $T T F 1, K R T 7$ and KRT19 expression could confirm the nonblood origin of the captured cells. The presence of KTR7 could be a sign of undifferentiated cell stages.
Table II. Results of the quantitative polymerase chain reaction analysis. A set of tumor-associated genes was tested in circulating tumor cells (CTCs)-enriched fractions. CTC fraction after short-term in vitro culture was compared to gene expression in the white blood cell fraction from whole blood. Genes with relatively higher RNA amount are marked $(X)$ in the column designated Elevated. Patient I: Suspicion of lung cancer; patient II: suspicion of pancreatic cancer.

\begin{tabular}{|c|c|c|c|c|}
\hline \multirow[b]{2}{*}{ Gene symbol (assay no.) } & \multicolumn{2}{|c|}{ Patient 1} & \multicolumn{2}{|c|}{ Patient 2} \\
\hline & $\begin{array}{l}\text { Gene } \\
\text { tested }\end{array}$ & $\begin{array}{c}\text { Gene } \\
\text { elevated }\end{array}$ & $\begin{array}{l}\text { Gene } \\
\text { tested }\end{array}$ & $\begin{array}{c}\text { Gene } \\
\text { elevated }\end{array}$ \\
\hline$A C T B$ (Hs01060665_g1) & $X$ & & $X$ & \\
\hline CD45 (Hs04189704_m1) & $\mathrm{X}$ & & $X$ & \\
\hline CD68 (Hs02836816_g1) & $\mathrm{X}$ & & $\mathrm{X}$ & \\
\hline EPCAM (Hs00158980_m1) & & & $X$ & $\mathrm{X}$ \\
\hline MUC1 (Hs00159357_m1) & $X$ & $\mathrm{X}$ & $X$ & \\
\hline TTF1 (Hs00201121_m1) & $\mathrm{X}$ & $X$ & & \\
\hline KRT5 (Hs00361185_m1) & $\mathrm{X}$ & & & \\
\hline KRT6 (Hs00603237_m1) & $\mathrm{X}$ & & & \\
\hline KRT7 (Hs00559840_m1) & $\mathrm{X}$ & $X$ & $X$ & $X$ \\
\hline KRT18 (Hs01920599_gH) & & & $X$ & $X$ \\
\hline KRT19 (Hs01051611_gH) & $X$ & $X$ & $\mathrm{X}$ & $\mathrm{X}$ \\
\hline KRT20 (Hs00300643_m1) & $\mathrm{X}$ & & $\mathrm{X}$ & \\
\hline VIM (Hs00185584_m1) & $\mathrm{X}$ & & & \\
\hline CD56 (Hs00941830_m1) & $X$ & & & \\
\hline CD57 (Hs00218629_m1) & $\mathrm{X}$ & & & \\
\hline SYP (Hs00300531_m1) & $\mathrm{X}$ & & & \\
\hline CEA (Hs00926316_m1) & $\mathrm{X}$ & & $X$ & \\
\hline Total $(\mathrm{N})$ & 15 & 4 & 10 & 4 \\
\hline
\end{tabular}

Finally, the patient underwent left upper lobectomy with subsequent resection of three ribs. Histopathological examination of the resected primary tumor confirmed lung adenocarcinoma.

Patient II. After cytomorphological analysis of captured cells on the separation membrane (Figure 3B) it was concluded that some of them exhibited cancerous characteristics (cell size $>15 \mu \mathrm{m}$, nucleus size $>10 \mu \mathrm{m}$, irregular nuclear contour). To confirm the non-blood cell origin of the captured cells, we analyzed expression of tumor-associated genes which were chosen due to the rules of differential diagnosis for pancreatic mass in pathology (ACTB, CD45, CD68, EpCAM, MUC1, KRT7, KRT18, KRT19, KRT20 and CEA). As reported in the Table II, EpCAM, KRT7, KRT18 and KRT19 were identified as being relatively highly expressed in comparison to the gene expression level in whole blood. It was concluded that the combination of KRT18 and KRT19 in combination with EpCAM expression confirmed the epithelial origin of the captured cells. KRT18 expression is typical for cells from the upper gastrointestinal tract. 
The patient underwent laparoscopy and disseminated tumor cells and tumors were detected in the peritoneal cavity (ascites, implanted metastasis on the mesenterium and peritoneum). The histopathological examination of resected metastases confirmed the diagnosis of pancreatic adenocarcinoma.

\section{Discussion}

The theory of the metastatic process is based on the fact that single tumor cells spread from the primary tumor either locally, leading to local metastases, or through lymphatic vessels to regional lymph nodes, or hematogenously in $\mathrm{PB}$ to settle in distant organs (1). Tumor cell dissemination is considered an early event in the multi-phasic metastatic process. The opportunity to detect tumor cells in the bloodstream before the clinical evidence of distant overt metastasis has been of interest for several decades (2-4). As we previously reported in our work, the presence of CTCs does not correspond with the TNM stage of disease, and for many tumor types, CTCs can be detected in early stages of disease (6-8). We found that $66.7 \%$ of patients with pancreatic cancer undergoing surgery had CTCs in their PB. (9). The presence of CTCs in the early stage of tumor disease opens up the possibility of using a CTC test not only to monitor cancer but also during diagnostic procedures.

The article reports on two cases of patients with suspicion of primary tumors in the lung and pancreas, but for whom it was not possible to specify the tumor mass histologically or cytologicaly before surgery. The histopathological specification of tumor before starting therapy is crucial and can significantly influence the treatment mode.

Modern medicine uses very precise endoscopic and scanning methods, however, even using these high-tech methods, in some cases it is not possible to confirm the tumor characteristics of cells. Especially in lung cancer, the histopathological classification may change the nature of the operation, which is why results of tumor classification should be provided immediately during surgery.

The focus of this study was to deliver information about the malignancy of the suspicious cell mass to the surgeon before planned surgery. We showed that the process of CTC testing may significantly help in cases where other methods of obtaining tumor tissue (e.g. tissue biopsy) are not possible.

CTCs are heterogenous and can differ between different cancer types, as well as within one patient over time. The heterogeneity of antigen expression and physical properties of tumor cells makes it quite challenging to identify the optimal approach for CTC selection and identification. EpCAM is widely used in many detection techniques to capture cells of epithelial origin. Both EpCAM-positive and EpCAM-negative CTCs can be present in one type of tumor and be influenced by treatment and disease stage (10). A size-based filter can exploit the antigen difference to isolate CTCs in a simple, fast and antibody-independent manner (5-9).
Both patients had subjective symptoms and changes identified during CT scanning. It was very difficult to obtain enough tumor tissue for histological verification of tumor presurgically. As reported, liquid biopsy via CTCs analysis could be helpful in these kind of complicated cases. Finally, in both cases, the presence of tumor cells was confirmed by postsurgical histopathology in concordance with our findings,

\section{Summary}

Detection of cancer cells in PB and their molecular characterization could significantly help to complete tumor diagnostic process in a time efficient manner.

\section{Acknowledgements}

This work was supported by internal grant Krajska zdravotni a.s. No. 217104001: Characterization of the Circulating Tumor Cell in Lung Cancer.

\section{References}

1 Budd GT, Cristofanilli M, Ellis MJ, Stopeck A, Borden E, Miller MC, Matera J, Repollet M, Doyle GV, Terstappen LW and Hayes DF: Circulating tumor cells versus imaging - predicting overall survival in metastatic breast cancer. Clin Cancer Res 12: 64036409, 2006.

2 Danila DC, Heller G, Gignac GA, Gonzalez-Espinoza R, Anand A, Tanaka E, Lilja H, Schwartz L, Larson S, Fleisher M and Scher HI: Circulating tumor cell number and prognosis in progressive castration-resistant prostate cancer. Clin Cancer Res 13: 7053-7058, 2007.

3 Hayes DF, Cristofanilli M, Budd GT, Ellis MJ, Stopeck A, Miller MC, Matera J, Allard WJ, Doyle GV and Terstappen LW: Circulating tumor cells at each follow-up time point during therapy of metastatic breast cancer patients predict progressionfree and overall survival. Clin Cancer Res 12: 4218-4224, 2006.

4 Shaffer DR, Leversha MA, Danila DC, Lin O, GonzalezEspinoza R, Gu B, Anand A, Smith K, Maslak P, Doyle GV, Terstappen LW, Lilja H, Heller G, Fleisher M and Scher HI: Circulating tumor cell analysis in patients with progressive castration-resistant prostate cancer. Clin Cancer Res 13: 20232029, 2007.

5 Liberko M, Kolostova $\mathrm{K}$ and Bobek V: Essentials of circulating tumor cells for clinical research and practice. Crit Rev Oncol Hematol 88: 338-335, 2013.

6 Kolostova K, Broul M, Schraml J, Cegan M, Matkowski R, Fiutowski M and Bobek V: Circulating tumor cells in localized prostate cancer: isolation, cultivation in vitro and relationship to T-stage and Gleason score. Anticancer Res 34: 3641-3646, 2014.

7 Bobek V, Matkowski R, Gürlich R, Grabowski K, Szelachowska J, Lischke R, Schützner J, Harustiak T, Pazdro A, Rzechonek A and Kolostova K: Cultivation of circulating tumor cells in esophageal cancer. Folia Histochem Cytobiol 52: 171-177, 2014.

8 Cegan M, Kolostova K, Matkowski R, Broul M, Schraml J, Fiutowski $\mathrm{M}$ and Bobek $\mathrm{V}$ : In vitro culturing of viable circulating tumor cells of urinary bladder cancer. Int J Clin Exp Pathol 7: 7164-7171, 2014. 
9 Bobek V, Gurlich R, Eliasova P and Kolostova K: Circulating tumor cells in pancreatic cancer patients: Enrichment and cultivation. World J Gastroenterol 20: 17163-17170, 2014.

10 van de Stolpe A and den Toonder JM: Circulating tumor cells: what is in it for the patient? A vision towards the future. Cancers 6: 1195-1207, 2014.

11 Cibes E and Ducatman B: Cytology. Diagnostic Principles and Clinical Correlates. Saunders, Elsevier, 2009.

12 Ting DT, Wittner BS, Ligorio M, Vincent Jordan N, Shah AM, Miyamoto DT, Aceto N, Bersani F, Brannigan BW, Xega K, Ciciliano JC, Zhu H, MacKenzie OC, Trautwein J, Arora KS, Shahid M, Ellis HL, Qu N, Bardeesy N, Rivera MN, Deshpande
V, Ferrone CR, Kapur R, Ramaswamy S, Shioda T, Toner M, Maheswaran S and Haber DA: Single-cell RNA sequencing identifies extracellular matrix gene expression by pancreatic circulating tumor cells. Cell Res 8: 1905-1918, 2014.

Received July 21, 2017

Revised August 21, 2017

Accepted September 4, 2017 